\title{
IPASC: a Community-Driven Consensus-Based Initiative Towards Standardisation in Photoacoustic Imaging
}

\author{
Sarah E. Bohndiek \\ University of Cambridge \\ Cambridge, U.K. \\ seb53@cam.ac.uk \\ Lina Hacker \\ University of Cambridge \\ Cambridge, U.K. \\ lh592@cam.ac.uk
}

\author{
Ben Cox \\ University College London \\ London, U.K. \\ b.cox@ucl.ac.uk
}

\author{
Stefan Morscher \\ iThera Medical GmbH \\ Munich, Germany \\ stefan.morscher@ithera-medical.com
}

\author{
James Joseph \\ University of Dundee \\ Dundee, U.K. \\ jjoseph001@dundee.ac.uk
}

\author{
Janek Gröhl \\ German Cancer Research Centre (DKFZ) \\ Heidelberg, Germany \\ j.groehl@dkfz-heidelberg.de
}

\author{
William C. Vogt \\ U.S. Food and Drug Administration (FDA) \\ Maryland, U.S.A. \\ william.vogt@fda.hhs.gov
}

\begin{abstract}
Photoacoustic Imaging (PAI) is a rapidly emerging imaging modality that is based on the conversion from light into ultrasound. One of the most promising applications of PAI is in diagnosis and longitudinal monitoring of solid tumours. By visualizing differences in the optical energy absorbed by endogenous chromophores, such as haemoglobin or melanin, PAI has made rapid advances over the past decade. In particular, PAI has been successfully demonstrated in early stage human trials in cancer, for delineating benign and malignant lesions as well as monitoring treatment.

The promise of these early pilot studies has led to the development of several commercial clinical PAI instruments. Furthermore, qualitative image interpretation is increasingly being replaced by calculation of quantitative imaging biomarkers (IBs) as both research tools and as putative clinical decisionmaking tools. Considering the recently reported roadmaps for clinical translation of IBs, it is clear that acceleration of PAI biomarkers into clinical use requires parallel technical, biological and clinical validation, as well as assessment of cost-effectiveness. The International Photoacoustic Standardisation Consortium (IPASC) has been founded to address this unmet need. The overall objective of IPASC is to reach an international consensus on PAI standardization to improve the quality of preclinical studies and to accelerate efforts in clinical translation.

Index Terms-photoacoustics, standardisation, phantoms
\end{abstract}

\section{INTRODUCTION}

Photoacoustic imaging (PAI) is an exciting technology that combines laser excitation with acoustic detection to enable deep-tissue imaging of absorbed optical energy, which can be used to visualise a range of biological properties, including vasculature and blood oxygenation [1]. PAI harnesses the photoacoustic effect, where absorbed optical energy is converted into acoustic waves detectable by ultrasound transducers. PAI provides images with spatial resolution determined by the ultrasound detection scheme but with strong contrast based on light absorption by molecules, such as haemoglobin found in blood, or exogenous contrast agents [2], [3]. Because sound is less attenuated in tissue than light, PAI can detect blood vessels and absorptive chromophores at high resolution much deeper than pure optical imaging (order of $\mathrm{cm}$ rather than $\mathrm{mm}$ ). As a result, PAI has been developed as a preclinical research tool for studying tissue oxygenation in diseases of inflammation and in cancer biology, as well as for monitoring drug biodistribution evaluation. PAI has also found utility in clinical applications including cancer detection (especially of breast cancer) [4], surgical guidance [5], tumour margin delineation [6], and cerebral oximetry [7]. Commercial systems are available for preclinical research, several clinical devices are currently marketed for investigational use, and two clinicalgrade devices have received the CE mark.

Despite the substantial growth of the PAI research community and significant investment by industry and government agencies, preclinical and clinical adoption of PAI has been slow. Notably, no devices have crossed the translational gap to applications in healthcare systems despite the strong potential of PAI for improving patient care. This is due in part to difficulties in evaluating system performance and comparing devices of different designs. Mature medical imaging modalities (MRI, CT, ultrasound) have internationally recognized standards that establish tools, test methods, and best practices for objective, quantitative image quality assessment [8]. They also have numerous commercial devices already approved for use as standard-of-care in a range of clinical indications. For PAI, no such standards or clinical devices yet exist due to its nascent status, placing a burden on device manufacturers to design their own test methods and potentially leading to duplication of effort and lack of community consensus. The availability of well-validated, consensus-based performance test methods for PAI systems would greatly accelerate device design optimization, device inter-comparison, quality assurance/control testing, and regulatory evaluation.

In recent years, substantial test methodology research has been conducted to help develop community consensus on 
best practices in testing PAI devices. Much of this research has focused on developing tissue-mimicking phantoms that replicate the optical and acoustic properties of tissues [9]-[11]. Phantoms provide low-cost, easily fabricated alternatives to in vivo animal testing, complying with principles of $3 \mathrm{Rs}$, and enable objective, quantitative benchtop assessment of device performance. Phantoms can be used to evaluate essential image quality metrics (e.g., resolution, contrast) [12]-[15] as well as performance of quantitative imaging biomarkers, such as total haemoglobin concentration, blood oxygen saturation, or exogenous contrast agent concentration [16], [17]. Phantoms can further be used to compare outputs of different imaging systems as well as to track their performance longitudinally over time, making them a cornerstone of reliable imaging.

IPASC was established in 2018 to bring together researchers, device developers, and government regulators to develop community-consensus on standardised PAI performance test methods. Standardisation has typically lagged behind in adoption of medical imaging technologies, often being initiated long after the first identification of critical challenges in evaluating safety and effectiveness of medical devices. Learning these lessons from our experiences of other medical imaging technologies, IPASC provides a route to 'jump start' such activities for PAI and develop consensus on how to assess PAI devices in order to ensure that device developers produce objectively safe, effective, and high-quality devices that improve patient care. Efforts to date have included agreement on terms and definitions for use in performance evaluation of PAI and developing best practices for multi-site phantom-based PAI device image quality comparison studies. A full list of the international membership of the consortium can be found on our website [18] and our organisational structure can be see in Figure 1.

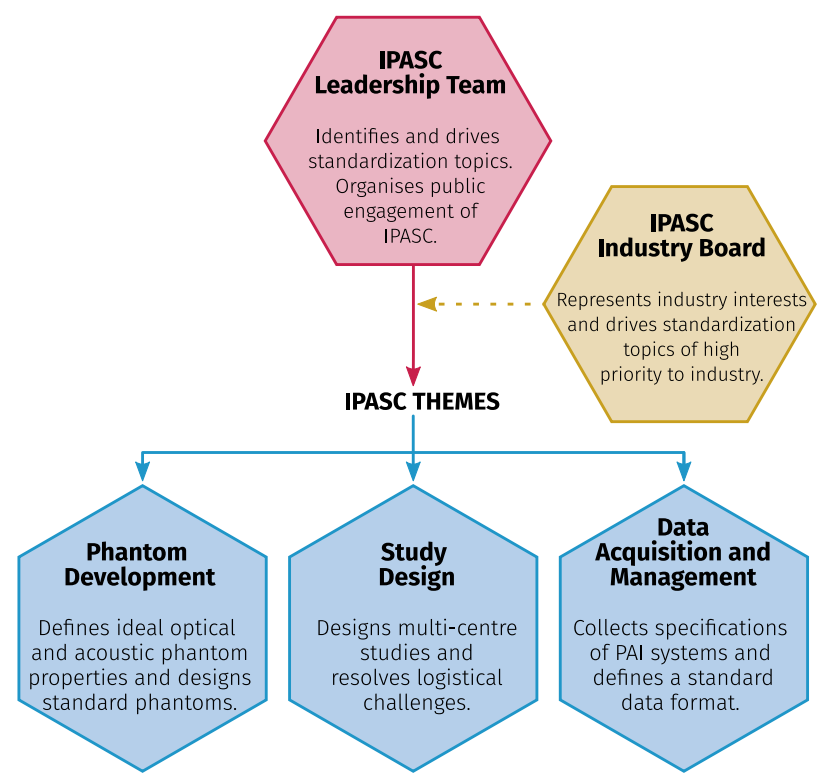

Fig. 1. IPASC organization into three thematic working groups as well as the IPASC leadership team and industry board.
The overall aims of IPASC are to:

1) Define widely accepted phantoms for use with preclinical and clinical PAI systems;

2) Use these defined phantoms to enable quantitative comparison of PAI data acquired with different system designs and analysed with different data reconstruction and spectral processing algorithms;

3) Provide open, publicly available, reference datasets for testing of data reconstruction and spectral processing algorithms in a widely accepted data format;

4) Agree upon standardized and validated test methods for new PAI instruments to aid comparison of published results.

\section{RECENT ACTIVITIES}

The activities of IPASC are pursued under three main themes: Phantom Development; Data Acquisition and Management; and Study Design. In addition, we have an industry board, representing the interests of our commercial vendor members.

\section{A. Phantom Development}

The Phantom Development (PD) theme of IPASC have led vital community efforts in developing phantom recipes suitable for PAI standardisation. In particular, initial work in the theme reviewed all available candidate materials for the production of stable test objects with appropriate optical and acoustic properties. The review identified an unmet need for a base phantom material where the components fulfil the essential requirements of a standard phantom (Figure 2) such as low-cost and scalability. A candidate polymer-in-oil based recipe was formulated to meet these demands and subjected to long-term testing for stability of optical, acoustic, and thermomechanical properties. Ease of fabrication was tested by distributing a basic recipe to over 16 receiver sites across the world, who then provided feedback on the local cost of materials and manufacturing process. A consensus document is currently being prepared to establish the essential technical requirements for the phantom material.

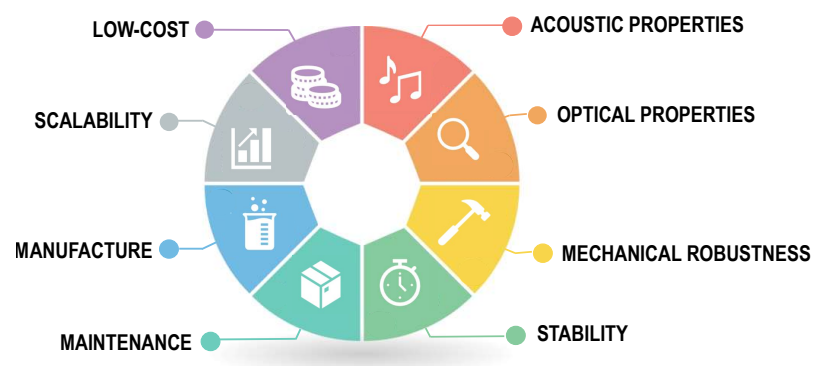

Fig. 2. Criteria that need to be fulfilled by a standard photoacoustic phantom.

\section{B. Data Acquisition and Management}

The efforts of the Data Acquisition and Management (DAM) theme have focused on establishing a standardized data 
format for photoacoustic time series data to facilitate interdevice data exchange and comparison. In order to achieve consensus on the data format, it was necessary to understand the full breadth of photoacoustic system geometries, including both illumination and detection elements, which was captured through a survey of the equipment available within our member laboratories. Furthermore, a detailed evaluation of all image metadata information was undertaken, considering the file container format, binary data metadata and acquisition metadata, which comprise the underpinning knowledge needed to reconstruct a photoacoustic image, as shown in Figure 3.

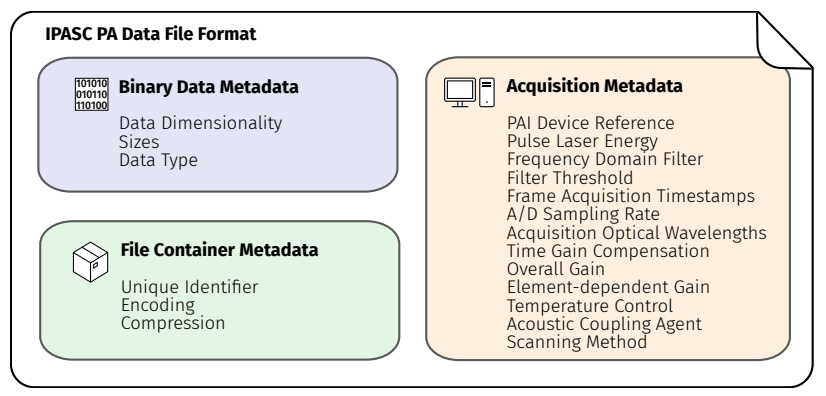

Fig. 3. Depiction of the three major components of the standard photoacoustic matedata to be reported for each photoacoustic image: (1) the file container metadata, (2) binary time series data, and (3) acquisition metadata.

The resulting consensus document [19] was adopted with only $5 \%$ of voters standing aside or rejecting the proposal and published openly for widespread community consultation on our website. Considering the requirements for photoacoustic data handling, the HDF5 file format has been identified as an appropriate data container and is the subject of ongoing work to develop a conversion tool between the existing file formats used by the community.

\section{Study Design}

Towards our goal of developing phantom-based test methods for PAI, the Study Design (SD) theme has undertaken consensus-building activities, including agreement of standardised terms and definitions used in the PAI community [20] as well as initial phantom testing guidelines and best practices. SD is currently organising consensus discussions to develop suitable phantom designs and image analysis techniques for evaluating fundamental image quality metrics that are necessary for characterisation of PAI system performance. An initial survey has been undertaken, the results of which were disseminated by video conference. Further discussion and debate within the consortium will lead to a draft consensus document containing recommended essential performance characteristics for PAI systems, agreed-upon phantom requirements (properties, geometry, target arrays) for testing these characteristics, and appropriate methods for computing image quality metrics from acquired data. The resulting document is intended to be completed by the end of 2020 .

\section{IPASC Industry Board}

The IPASC Industry board was inaugurated in 2020. It represents industry-specific interests in the context of stan- dardization and consists of vendors of photoacoustic imaging equipment or components. Its purpose is to drive aspects of standardization that are specifically relevant to members of industry, but of lesser interest to the scientific research community. As its first work-item, the Industry Board initiated the inauguration of a Digital Imaging and Communications in Medicine (DICOM) working group (WG-34) with the goal to extend the widely used standard for clinical image management and transfer with support for PAI. As such, voting members from the industry are warmly welcomed to join both the Industry Board as well as the DICOM WG-34.

\section{FUTURE WORK}

Ongoing work in the PD and SD themes will culminate in a set of consensus recommendations establishing best practices for objective, quantitative assessment of PAI device image quality through phantom-based performance test methods. IPASC intends to demonstrate utility of these phantom-based test methods through a multi-site phantom imaging study among members, which is planned for 2021. Participating members will receive a phantom constructed following IPASC guidelines, perform a battery of image quality experiments and data analysis following an IPASC-developed test method, and submit results for comparison across different PAI systems. Study results are expected to demonstrate utility of phantombased image quality testing and promote acceptance of IPASC test methods, which will facilitate device development and optimization, inter-system comparison, and clinical translation. In parallel, the DAM theme is implementing a Python tool for conversion of existing file formats into the proposed standardised HDF5-based file format with the overall aim of using this format to launch a common and open-source data platform in 2021. Future goals include evolving current communityled consensus-based activities into broader standardisation efforts through formal standards working groups, such as the aforementioned DICOM working group for PAI. Prospective new members are invited to join our initiative via our website [18].

\section{ACKNOWLEDGMENT}

IPASC would like to thank all its members for their valuable contributions to the ongoing activities, particularly the consensus finding discussions.

The mention of commercial products, their sources, or their use in connection with material reported herein is not to be construed as either an actual or implied endorsement of such products by the US Department of Health and Human Services. This article reflects the views of the authors and should not be construed to represent FDA views or policies.

\section{REFERENCES}

[1] Wang, L.V. and S. Hu, Science. 335(6075). 1458-62 (2012)

[2] Lemaster, J.E. and J.V. Jokerst, Wiley Interdiscip Rev Nanomed Nanobiotechnol. 9(1). (2017)

[3] Weber, J., P.C. Beard, and S.E. Bohndiek, Nat Methods. 13(8). 639-50 (2016).

[4] Manohar, S. and M. Dantuma, Photoacoustics. 16. 100134. (2019)

[5] Lediju Bell, M.A., et al., Journal of Biomedical Optics. 22(12). 1 (2017) 
[6] Li, R., et al., Biomed Opt Express. 6(4). 1273-81 (2015)

[7] Petrova, I.Y., et al., Opt Express. 17(9). 7285-94 (2009)

[8] Pfefer, J. and A. Agrawal, Proc. of SPIE. 8215(82150D). 82150D-1-10 (2012)

[9] Vogt, W.C., et al., J Biomed Opt. 21(10). 101405 (2016)

[10] Maneas, E., et al., Biomed Opt Express. 9(3). 1151-1163 (2018)

[11] Bohndiek, S.E., et al., PLoS One. 8(9). e75533 (2013)

[12] Ratto, F., et al., Biomed Opt Express. 10(8). 3719-3730 (2019)

[13] Vogt, W.C., et al., J Biomed Opt. 22(9). 1-14 (2017)

[14] Joseph, J., et al., J Nucl Med. 58(5). 807-814 (2017)

[15] Arconada-Alvarez, S.J., et al., Photoacoustics. 5 17-24 (2017)

[16] Gehrung, M., S.E. Bohndiek, and J. Brunker, J Biomed Opt. 24(12). 1-11 (2019)

[17] Vogt, W.C., et al., Biomed Opt Express. 10(2). 449-464 (2019)

[18] www.ipasc.science, accessed August 5th 2020.

[19] www.ipasc.science/documents/20200121_Metadata list.pdf, accessed August 5th 2020.

[20] www.ipasc.science/documents/20191210_Terms_Definitions_For_PAT. pdf, accessed August 5th 2020. 